\title{
Metformin Is Associated With Survival Benefit in Cancer Patients With Concurrent Type 2 Diabetes: A Systematic Review and Meta-Analysis
}

\author{
Ming Yin, ${ }^{a}$ Jie ZHOU, ${ }^{b}$ EDWARD J. GoraK, ${ }^{c}$ FAHD QUdDUS ${ }^{c}$ \\ ${ }^{a}$ Department of Internal Medicine, ${ }^{b}$ Department of Radiology, and ${ }^{c}$ Department of Hematology and Oncology, Geisinger Medical Center, \\ Danville, Pennsylvania, USA \\ Disclosures of potential conflicts of interest may be found at the end of this article.
}

Key Words. Metformin • Cancer • Diabetes • Survival • Meta-Analysis

Learning Objectives Describe the influences of different types of glucose-lowering medications on therapeutic outcomes of cancer patients who received standard anticancer treatment.

Compare the survival associated with metformin treatment with survival in treatment with other glucose-lowering medications.

\begin{abstract}
Purpose. Patients with type 2 diabetes have increased cancer risk and cancer-related mortality, which can be reduced by metformin treatment. However, it is unclear whether metformin can also modulate clinical outcomes in patients with cancer and concurrent type 2 diabetes.

Patients and Methods. A meta-analysis of 20 publications that included 13,008 subjects was performed to investigate the association between metformin and overall survival (OS) as well as cancer-specific survival (CSS) in patients with cancer and concurrent type 2 diabetes.
\end{abstract}

Results. We found that there was a relative survival benefit associated with metformin treatment compared with treatment with other glucose-lowering medications in both OS and CSS (hazard ratio $[\mathrm{HR}]=0.66 ; 95 \%$ confidence interval $[\mathrm{Cl}]$ : $0.55-0.79$ and $\mathrm{HR}=0.62 ; 95 \% \mathrm{Cl}: 0.46-0.84$, respectively). These associations were also observed in subgroups by cancer type and country.

Conclusion. These results suggest that metformin is the drug of choice in the treatment of patients with cancer and concurrent type 2 diabetes. The Oncologist 2013;18:1248-1255

Implications for Practice: Patients with type 2 diabetes have increased cancer risk and cancer-related mortality, which can be reduced by metformin treatment. However, it is unclear whether metformin can also modulate clinical outcomes in patients with cancer and concurrent type 2 diabetes. Our meta-analysis provided evidence that there was a relative survival benefit associated with metformin treatment compared with treatment with other glucose-lowering medications. Our results suggest that metformin is the drug of choice in the treatment of patients with cancer and concurrent type 2 diabetes.

\section{INTRODUCTION}

Patients with cancer and diabetes mellitus are frequently encountered in clinical practice. It is estimated that approximately $8 \%$ to $18 \%$ of people with cancer have concurrent diabetes, probably because of the shared risk factors between the diseases and their increasing global prevalence [1]. There is epidemiologic evidence supporting a biologic link between cancer and type 2 diabetes, and there is a significantly higher cancer incidence and cancer-related mortality in those with diabetes. Plausible mechanisms of the increased carcinogenesis and neoplastic proliferation in those with diabetes could be multifactorial, including the effect of hyperinsulinemia, hyperglycemia, and inflammatory cytokines produced by adipose tissues [2]. It is conceivable that selection of glucose- controlling agents without the above pathophysiologic effects may protect against cancer development.

Metformin is one of the most commonly prescribed medications for the management of type 2 diabetes mellitus. Although its exact mechanism of action is not fully understood, metformin treatment inhibits hepatic gluconeogenesis [3, 4], reduces insulin resistance [5], improves glycemic control, and decreases inflammatory response $[6,7]$, thus avoiding the potential tumor-promoting effect. Some in vitro and in vivo studies further demonstrated that metformin might have direct antitumor activity by inhibiting cancer cell proliferation and colony formation [8, $9]$, inducing cell cycle arrest and apoptosis $[10,11]$ and suppressing xenograft tumor growth in mouse models $[12,13]$. All these

Correspondence: Ming Yin, M.D., Department of Internal Medicine, Geisinger Medical Center, 100 North Academic Avenue, Danville, Pennsylvania 17822, USA. Telephone: 570-271-6164; Fax: 570-271-6141; E-Mail: myin@geisinger.edu; or Fahd Quddus, M.D., Department of Hematology and Oncology, Geisinger Medical Center, 100 North Academic Avenue, Danville, Pennsylvania 17822, USA. Telephone: 864-255-1826; E-Mail: fquddus@geisinger.edu Received March 30,2013; accepted for publication September 12, 2013; first published online in The Oncologist Express on November 20, 2013. CAlphaMed Press 1083-7159/2013/\$20.00/0 http://dx.doi.org/10.1634/theoncologist.2013-0111 
characteristics make metformin an ideal drug for cancer prevention compared with insulin and insulin secretagogues in type 2 diabetes treatment. Indeed, results of human observational studies and meta-analyses suggest that treatment with metformin is associated with reduced cancer risk or cancer mortality compared with other glucose-lowering therapies $[14,15]$.

Although metformin seems to be a promising cancer preventive medication, it is not clear whether it is also effective in those with type 2 diabetes and an established cancer diagnosis. Since 2009, a growing number of studies have compared metformin versus non-metformin treatment on survival outcomes of those with diabetes in various types of malignancies, including breast, colorectal, prostate, lung, hepatic, and ovarian cancer. Because published reports from individual studies are not consistent [16, 17], we performed a systematic review and meta-analysis to assess the association between metformin usage and survival outcome of patients with concurrent diabetes and cancer.

\section{Patients And Methods}

\section{Study Selection}

We searched Medline and PubMed databases for relevant articles published as of July 1, 2013, in English-language literature using the following terms: metformin, diabetes, cancer, tumor, and survival. References of the retrieved articles were further screened for earlier original studies. The inclusion criteria were as follows: solid cancer, cancer and concurrent diabetes, cancer histologically or pathologically confirmed, and overall survival (OS) or cancer-specific survival (CSS) reported for metformin- and non-metformin-treated patients. The corresponding authors were contacted to obtain missing information. Abstracts, unpublished reports, and articles not written in English were excluded.

\section{Data Extraction}

We extracted the following information from each published article: author, year of publication, country of origin, cancer type, ethnicity, sample size, glucose-lowering medications given, and follow-up period. We used adjusted Cox proportional hazard ratios (HRs) for the quantitative analysis. If adjusted HRs were not available and the corresponding authors did not respond to our request, the crude HRs were used. When both adjusted and crude HR data were not available but appropriate summary statistics or Kaplan-Meier curves were provided, we calculated HRs and 95\% confidence intervals (Cls) as relevant effect measures using published methods.

\section{Statistical Methods}

OS and CSS were evaluated by pooled Cox proportional HRS and $95 \% \mathrm{Cls}$ were evaluated using published methods [18] because a meta-analysis of summary results is statistically as efficient as a joint analysis of individual participant data [19]. We assessed the between-study heterogeneity by using the Cochran $Q$ test with a significance level of $p<.10$. We performed initial analyses with a fixed-effect model and confirmatory analyses were done with a random-effect model if there was significant heterogeneity. We used inverted funnel plots and the Egger's test to examine the effect of publication bias. A "trim and fill" method was used to make adjusted estimations in the presence of publication bias [20]. All $p$ values were twosided, and all analyses were performed using the Stata software (StataCorp, College Station, TX, USA http://www.stata. com) and Review Manager (v5.0; Oxford, United Kingdom http://ims.cochrane.org/revman)

\section{ReSUlTS}

\section{Meta-Analysis Database}

Figure 1 shows the flow chart of study selection. We identified 210 related publications by initial screening, of which 25 publications seemed to meet the inclusion criteria. We excluded three studies [21-23] in which no survival information was reported or survival information was not obtainable. We further excluded the study by Lee et al. because it was not written in English [24] and the study by Koo et al. because it was not conducted in solid cancer and did not provide survival information in those with lymphoma and diabetes [25]. The study by Kumar et al. was included for analysis of CSS because it reported only CSS and not OS [26]. As a result, the final data pool consisted of 20 studies, including 13,008 patients with cancer and concurrent type 2 diabetes (Table 1). Among the 20 studies, OS information was available in 19 studies and CSS information was available in 9 studies. Among the 13,008 patients, 6,343 patients received metformin alone or in combination with other glucose-lowering regimens and the remaining 6,665 patients received non-metformin treatment such as insulin, sulfonylurea, thiazolidinedione, alpha-glucosidase inhibitor, dipeptidyl peptidase- 4 inhibitors, amylin analogs, or glucagon-like peptide-1 analogs.

\section{Quantitative Analyses}

\section{Overall Survival}

As summarized in Figure 2, 19 of the 20 selected articles reported OS. The exception was the study by Kumar et al.

In a fixed model analysis stratified by cancer type, we found that metformin was associated with a significantly reduced risk for death in those with breast, prostate, pancreatic, colorectal, and other cancers, with the exception of lung cancer. In random model analysis, metformin was associated with a significantly reduced risk for death in those with pancreatic, colorectal, and other cancers, and with a nonsignificantly reduced death risk in those with lung, breast, and prostate cancer.

which reported only CSS. Our meta-analysis demonstrated that the administration of metformin to patients with cancer and type 2 diabetes was associated with significantly reduced risk for death compared with those who did not receive metformin $(\mathrm{HR}=0.66 ; 95 \% \mathrm{Cl}: 0.55-0.79$ by random effect; $p<.001$ for heterogeneity; $l^{2}=83 \%$ ) (Table 2 ). The study by Currie et al. included $39.1 \%$ of the total patient population $(5,016 / 12,844)$ [27], which might dominate the analysis, and therefore, we performed sensitivity analyses by excluding one study at a time. After exclusion of the study by Currie et al. , the pooled HR was not significantly changed ( $\mathrm{HR}=0.65 ; 95 \% \mathrm{Cl}: 0.53-0.79$ by random effect; $p<.001$ for heterogeneity; $\left.I^{2}=83 \%\right)$. No single study influenced the pooled HRs qualitatively.

Heterogeneity analysis showed that there was substantial between-study heterogeneity among the 19 studies 


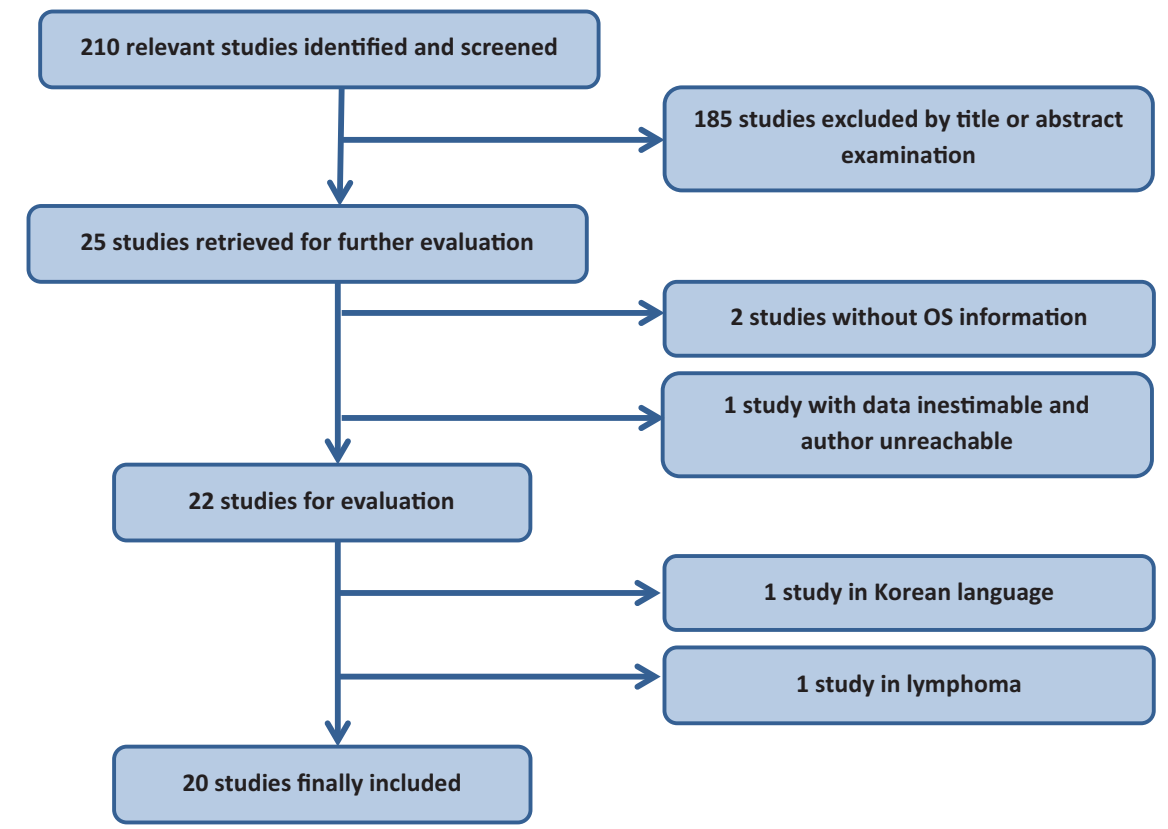

Figure 1. Study flow chart for the process of selecting the final 20 publications.

Abbreviations: OS, overall survival.

Table 1. Characteristics of the studies included in the meta-analysis

\begin{tabular}{|c|c|c|c|c|c|c|c|c|}
\hline Study & Year & Ethnicity & Country & $n$ (met/non-met) & Cancer & OS & CSS & Median follow-up \\
\hline Sadeghi et al. [30] & 2012 & Mixed & USA & $302(117 / 185)$ & Pancreatic & $\mathrm{Y}$ & $\mathrm{N}$ & 11.4 months \\
\hline Garrett et al. [31] & 2012 & Mixed & UK & $424(208 / 216)$ & Colorectal & $\mathrm{Y}$ & $\mathrm{N}$ & 70.7 months \\
\hline Currie et al. [27] & 2012 & Mixed & UK & $5016(2843 / 2173)$ & Mixed & $Y$ & $\mathrm{~N}$ & 19.2 months \\
\hline Romero et al. [32] & 2012 & Mixed & USA & $44(16 / 28)$ & Ovarian & $Y$ & $\mathrm{~N}$ & 63 months \\
\hline He et al. [33] & 2012 & Mixed & USA & $154(88 / 66)$ & Breast & Y & Y & 47.6 months \\
\hline Lee et al. [34] & 2011 & Asian & Korea & $595(258 / 337)$ & Colorectal & Y & Y & 41 months \\
\hline Bayraktar et al. [35] & 2012 & Mixed & USA & $130(63 / 67)$ & Breast & $\mathrm{Y}$ & Y & 62 months \\
\hline Tan et al. [17] & 2011 & Asian & China & $99(39 / 60)$ & NSCLC & Y & $\mathrm{N}$ & N/A \\
\hline He et al. [36] & 2011 & Mixed & USA & $233(132 / 101)$ & Prostate & $Y$ & $\mathrm{~N}$ & N/A \\
\hline Chen et al. [37] & 2011 & Asian & Taiwan & $53(21 / 32)$ & Liver & $Y$ & $\mathrm{~N}$ & 32.2 months \\
\hline Nakai et al. [38] & 2012 & Asian & Japan & $124(8 / 116)$ & Pancreatic & $\mathrm{Y}$ & $\mathrm{N}$ & 9.9 months \\
\hline Mazzone et al. [16] & 2012 & Mixed & USA & $522(184 / 338)$ & NSCLC/SCLC & Y & $\mathrm{N}$ & N/A \\
\hline Spratt et al. [39] & 2012 & Mixed & USA & $319(157 / 162)$ & Prostate & $\mathrm{Y}$ & $Y$ & 8.7 years \\
\hline Hou et al. [40] & 2013 & Asian & China & $1013(419 / 594)$ & Breast & Y & $\mathrm{N}$ & 68 months \\
\hline Kumar et al. [26] & 2012 & Mixed & USA & $164(61 / 103)$ & Ovarian & $\mathrm{N}$ & Y & 11 years \\
\hline Spillance et al. [41] & 2013 & White & Ireland & $315(207 / 108)$ & Colorectal & Y & Y & N/A \\
\hline Cossor et al. [42] & 2013 & Mixed & USA & $212(84 / 128)$ & Colorectal & Y & $\mathrm{Y}$ & 4.1 years \\
\hline Kaushik et al. [28] & 2013 & Mixed & USA & $885(323 / 562)$ & Prostate & Y & $\mathrm{Y}$ & 5.1 years \\
\hline Lega et al. [29] & 2013 & Mixed & Canada & $2361(1094 / 1267)$ & Breast & $\mathrm{Y}$ & $\mathrm{Y}$ & 4.5 years \\
\hline Sandulache et al. [43] & 2013 & Mixed & USA & $43(21 / 22)$ & Larynx & Y & $\mathrm{N}$ & N/A \\
\hline
\end{tabular}

Abbreviations: CSS, cancer-specific survival; met, metformin; non-met, non-metformin; N/A, not available; NSCLC, non-small cell lung cancer; OS, overall survival; SCLC, small cell lung cancer.

( $p<.001$ for heterogeneity; $l^{2}=83 \%$ ). The between-study heterogeneity mainly resulted from the three studies by Mazzone et al. [16], Kaushik et al. [28], and Lega et al. [29], exclusion of which did not change the corresponding pooled HR substantially (metformin vs. non-metformin: $\mathrm{HR}=0.60 ; 95 \% \mathrm{Cl}: 0.55-0.66$ by fixed effects; $p=.173$ for heterogeneity; $I^{2}=25 \%$ ). We further performed funnel plot (Fig. 3) and Egger's test ( $p=.008)$, which suggested the possible presence of publication bias. Using a "trim and fill" method to make an adjusted estimation of meta-analysis, we found that metformin was still associated with reduced death risk in the adjusted analyses $(\mathrm{HR}=0.89 ; 95 \% \mathrm{Cl}$ : $0.86-0.94$ by fixed effects, and $\mathrm{HR}=0.74 ; 95 \% \mathrm{Cl}: 0.62-$ 0.87 by random effects). 


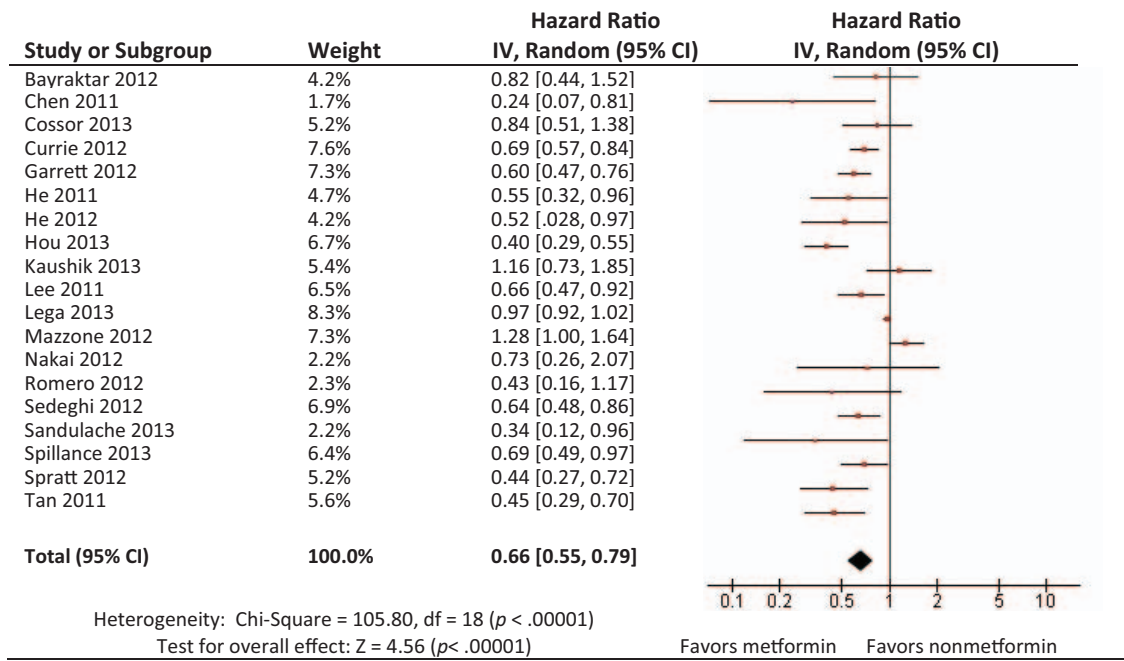

Figure 2. Hazard ratios (HRs) of overall survival by metformin versus non-metformin exposure. For each study, the estimates of $\mathrm{HR}$ and $95 \% \mathrm{Cl}$ were plotted with a box and a horizontal line. Closed diamond indicates pooled $\mathrm{HR}$ and $95 \% \mathrm{Cl}$.

Abbreviations: $\mathrm{Cl}$, confidence interval; $\mathrm{df}$, degrees of freedom.

Table 2. Associations between metformin and overall survival

\begin{tabular}{lclll}
\hline & & \multicolumn{3}{c}{ HR (95\% Cl) } \\
\cline { 3 - 4 } Variables & Sample Size & Fixed & Random & phet \\
\hline All cancer & 12,829 & $0.88(0.85-0.92)$ & $0.66(0.55-0.79)$ & \\
Cancer site & & & $0.77(0.28-2.15)$ & $<.001$ \\
Lung & 606 & $1.00(0.81-1.24)$ & $0.64(0.37-1.12)$ & $<.001$ \\
Breast & 3,658 & $0.94(0.90-0.99)$ & $0.66(0.36-1.21)$ & .012 \\
Prostate & 1,437 & $0.68(0.51-0.90)$ & $0.65(0.49-0.86)$ & .813 \\
Pancreatic & 426 & $0.65(0.49-0.86)$ & $0.65(0.56-0.77)$ & .662 \\
Colorectal & 1,546 & $0.65(0.56-0.77)$ & $0.34(0.18-0.63)$ & .767 \\
Other & 140 & $0.34(0.18-0.63)$ & & .151 \\
Country & & & $0.49(0.37-0.65)$ & $<.001$ \\
Asian & 1,884 & $0.49(0.40-0.60)$ & $0.73(0.61-0.87)$ \\
Western & 10,945 & $0.91(0.87-0.95)$ & & \\
\hline
\end{tabular}

Abbreviations: $\mathrm{Cl}$, confidence interval; $\mathrm{HR}$, hazard ratio; $p$ het: $p$ value of the $Q$ test for heterogeneity.

\section{Stratified Analyses}

In a fixed model analysis stratified by cancer type, we found that metformin was associated with a significantly reduced risk for death in those with breast, prostate, pancreatic, colorectal, and other cancers, with the exception of lung cancer. In a random model analysis, metformin was associated with a significantly reduced risk for death in those with pancreatic, colorectal, and other cancers, and with a nonsignificantly reduced death risk in those with lung, breast, and prostate cancer (Table 2).

We next performed subgroup analyses by country (Asian or Western countries). In the subgroup of Asian countries, metformin was still associated with reduced death risk (HR $=$ $0.49 ; 95 \% \mathrm{Cl}: 0.40-0.60$ by fixed effect; $p=.150$ for heterogeneity; $I^{2}=41 \%$ ). In the subgroup of Western countries, the relative survival benefit associated with metformin remained $(\mathrm{HR}=0.73 ; 95 \% \mathrm{Cl}: 0.61-0.87$ by random effect; $p<.001$ for heterogeneity; $\left.I^{2}=79 \%\right)$. No publication bias was detected in the stratified analyses (data not shown).
Cancer-Specific Survival A total of nine studies reported CSS (Table 1). Our meta-analysis showed that metformin administration was significantly associated with reduced cancerrelated mortality compared with not receiving metformin $(\mathrm{HR}=0.62 ; 95 \% \mathrm{Cl}: 0.46-0.84$ by random effect; $p<.001$ for heterogeneity; $l^{2}=79 \%$ ). Similar results were also obtained in stratified analysis by cancer type (Table 3). Stratified analyses by ethnicity were not performed because there were insufficient data from Asian countries. No publication bias was detected in either the overall study analysis or the stratified analysis (data not shown).

\section{DisCUSSION}

To our knowledge, this is the first and the largest quantitative analysis to examine the association between metformin treatment and clinical outcomes of patients with concurrent cancer and type 2 diabetes. In this meta-analysis, including 13,008 patients from 20 independently published investigations, we provided evidence that use of metformin was associated with 


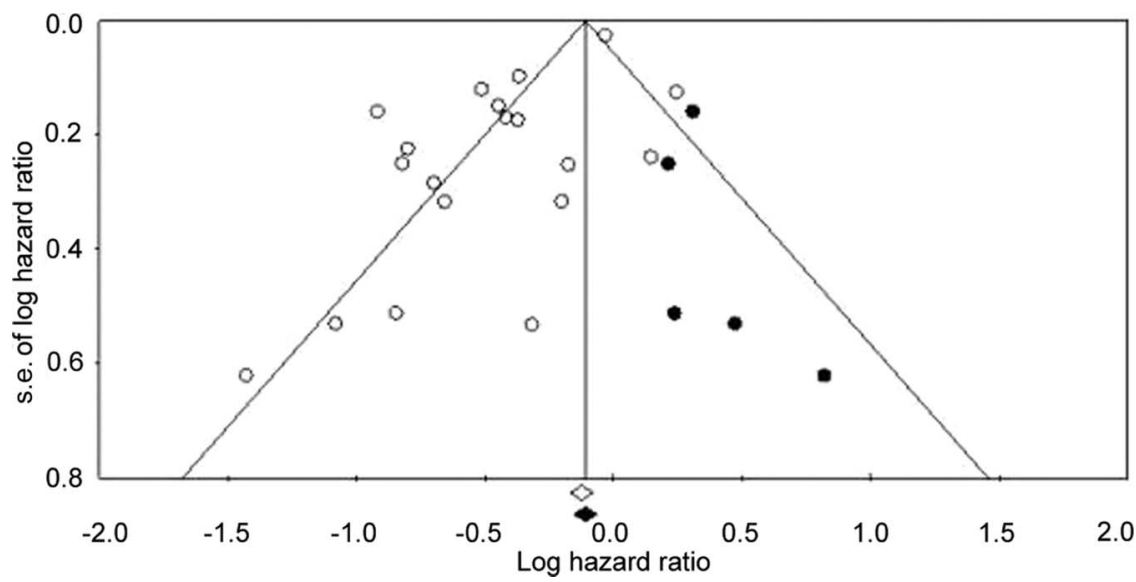

Figure 3. Funnel plot analysis to detect publication bias. Each circle represents an individual study for the indicated association. Hollow circles indicate original observation studies; solid circles indicate missing studies by trim and fill method.

Abbreviation: s.e., standard error.

Table 3. Associations between metformin and cancer-specific survival

\begin{tabular}{lllll}
\hline & & \multicolumn{3}{c}{ HR (95\% Cl) } \\
\cline { 3 - 4 } Variables & $\boldsymbol{n}$ & Fixed & Random & phet \\
\hline All cancer & 5,135 & $0.77(0.70-0.85)$ & $0.62(0.46-0.84)$ & \\
Cancer site & & & $0.76(0.53-1.08)$ & .001 \\
Breast & 2,645 & $0.88(0.79-0.99)$ & $0.66(0.50-0.87)$ & .878 \\
Colorectal & 1,122 & $0.66(0.50-0.87)$ & $0.47(0.27-0.81)$ & .073 \\
Other & 1,368 & $0.40(0.30-0.53)$ &
\end{tabular}

Abbreviations: $\mathrm{Cl}$, confidence interval; $\mathrm{HR}$, hazard ratio; $p$ het: $p$ value of the $Q$ test for heterogeneity.

improved OS and CSS in the treatment of patients with type 2 diabetes and cancer.

Cancer and diabetes are the second and seventh leading causes of death in the United States. Type 2 diabetes is the most common form of diabetes mellitus; more than $90 \%$ of those with diabetes have type 2 diabetes. Certain types of cancer were found to occur more commonly in those with diabetes, such as liver, pancreatic, breast, colorectal, bladder, and endometrial cancer. Prostate cancer seemed to be the exception, occurring less often in individuals with type 2 diabetes [44]. The biologic link between cancer risk and type 2 diabetes seemed to be influenced by different types of glucose-lowering agents. For example, insulin and insulin secretagogues such as sulfonylurea can theoretically stimulate tumor development by activating insulin and insulin-like growth factor-1 receptor through hyperinsulinemia. Insulin-sensitizing peroxisome proliferator-activated receptor agonists such as thiazolidinediones do not increase insulin secretion directly, but were found to potentiate tumorigenesis in rodent studies [45]. In contrast, metformin does not carry any inherent tumor-promoting characteristics and showed antitumor activities in a substantial number of studies both in vitro and in vivo. These studies suggested that metformin has multiple tumorsuppressing mechanisms, including potentiating other antineoplastic agents by downregulation of p38 mitogenactivated protein kinase signaling $[46,47]$, inhibition of protein synthesis by $5^{\prime}$ adenosine monophosphate-activated protein kinase (AMPK)-dependent and AMPK-independent pathways [3, 48-50], and alteration of energy metabolism by modulating mircoRNA through Dicer and c-myc [51]. Consistent with these findings, human observational studies and related meta-analyses confirmed that metformin reduced

A cancer-preventive advantage associated with metformin does not necessarily imply effective therapeutic efficacy in those with diabetes and established cancer. It is unclear whether the use of metformin could also translate into better clinical outcomes for patients with cancer who also receive standard cancer therapy.

cancer incidence and cancer-related mortality in those with type 2 diabetes compared with sulfonylureas and exogenous insulin $[15,52]$. However, a cancer-preventive advantage associated with metformin does not necessarily imply effective therapeutic efficacy in those with diabetes and established cancer. It is unclear whether the use of metformin could also translate into better clinical outcomes for patients with cancer who also receive standard cancer therapy.

Since 2009 , a series of retrospective investigations has reported that metformin might modulate the clinical outcomes of patients with cancer. Jiralerspong et al. reported a threefold greater pathologic complete response rate in patients with diabetes and breast cancer who received metformin during neoadjuvant chemotherapy compared with those who did not receive metformin (odds ratio: $2.95 ; 95 \% \mathrm{Cl}, 1.07-8.17$ ) [23]. 
Several other studies compared survival outcomes between metformin and non-metformin treatment in patients with cancer regardless of diabetes status, showing a better survival in the group who received metformin [34]. More recently, Kumar et al. reported that metformin was associated with significantly increased CSS in a cohort of 215 patients with ovarian cancer who did and did not have diabetes and in a cohort of 164 patients with epithelial ovarian cancer and concurrent type 2 diabetes (5-year CSS: $67 \%$ [metformin] vs. $43 \%$ [insulin] vs. $34 \%$ [other medications]; $p=.004$ ) [26]. In the current study, we included only patients with concurrent cancer and type 2 diabetes to highlight the impact of different antidiabetic medications on cancer prognosis, without involving diabetes itself as a confounder. We selected OS and CSS as study endpoints because OS has been the gold standard for demonstrating clinical benefit of metformin treatment, and CSS can provide evidence of therapeutic efficacy of metformin in cancer treatment.

A total of 13,008 patients with cancer and concurrent type 2 diabetes were included in the meta-analysis. Cancer types included pancreatic, colorectal, ovarian, breast, prostate, bladder, liver, larynx, and lung cancer. Compared with non-metformin treatment, metformin treatment carries a relative OS benefit in patients with concurrent cancer and type 2 diabetes, with a $34 \%$ reduction of death risk in the metformin-treated group ( $\mathrm{HR}=$ 0.66; 95\% Cl: 0.55-0.79). In stratified analyses by cancer type, metformin was associated with a significantly reduced death risk for those with pancreatic, colorectal, and other cancers, and a nonsignificantly reduced death risk for those with lung, breast, and prostate cancer. In stratified analyses by country (Asian or Western countries), the survival benefit remained. Similar results were obtained in CSS analyses. It is possible that the observed survival benefit in overall cancer is the result of differences in the types of cancer between groups, such as a higher proportion of pancreatic cancer (a poor prognosis tumor) in the non-metformin group versus metformin group. Although we cannot determine the exact proportion of patients with pancreatic cancer between groups because of a lack of related information in the study by Currie et al. [27], such a possibility was not supported because the survival benefit associated with metformin remained in pancreatic cancer in subgroup analysis, and including or excluding pancreatic cancer did not significantly change our analysis results in overall cancer. We were unable to perform stratified analyses by ethnicity (Asian or white) because related data were unavailable. However, stratified analysis by country could be used as a rough estimation of stratification by ethnicity because the majority of patient populations in the studies of Western countries were white, whereas the other studies included patients of pure East Asian ethnicity (China, Taiwan, Japan, and Korea).

It is important to note that the study by Mazzone et al. is the only study showing a significantly increased risk for death associated with metformin treatment [16], whereas most of the studies in our data pool, including the other study performed in lung cancer by Tan et al. [17], favor a protective effect of metformin. Several possibilities might explain the discrepancy. First, some other confounding factors (e.g., comorbidities, tumor histology) might exist and bias the analysis results in the study by Mazzone et al. As an example, there were more patients with metastatic cancer in the metformin-treated group compared with the nonmetformin-treated group. In addition, the study by Tan et al. included only patients with advanced non-small cell lung cancer who received chemotherapy, whereas the study by Mazzone et al. included patients with both small cell and non-small cell lung cancer irrespective of tumor stage and treatment received. It is possible that the effect of metformin could be tumor specific, and there might be unknown interactions between metformin and lung cancer treatment that influenced clinical outcomes of these patients. Therefore, the associated death risks in the two studies could both be true findings. Further investigations are required to address this issue.

Despite our efforts to provide an accurate and comprehensive analysis, limitations of our meta-analysis need to be addressed. First, some data were excluded from our analysis, which could cause some bias in our estimates, but was unlikely to change our major conclusions because nearly all of the excluded studies favored a better clinical outcome associated with metformin treatment [22-24], which was consistent with our findings. Second, most of the included studies were retrospective and differed significantly in study design, and additionally, our analysis largely used adjusted estimates, which were not adjusted by the same potential confounders in the published studies. We also used unadjusted estimates for the studies by Tan et al. and Hou et al. $[17,40]$ because the authors did not respond to our data request. All of these factors may have caused wide and significant heterogeneity among studies. However, when only unadjusted estimates were used, the conclusions were not significantly changed (data not shown). Third, both the tumor-suppressing activities of metformin and the tumor-promoting effect of other diabetic medications may contribute to the relative survival benefit of metformin observed in the current study. However, we were unable to determine the absolute survival benefit because related data were lacking. Fourth, dividing patients based on metformin exposure versus non-exposure may be too simple. Most patients with diabetes are treated with one or more glucose-lowering medications, with changes in pharmacotherapy over time. It is extremely difficult to determine the minimum level of exposure (time and dosage) required to observe the survival benefit with metformin. $A$ possible method would be to explore the dose-response relationship in metformin users; however, few studies provided this information. Finally, there seemed to be significant publication bias in the literature, as suggested by the funnel plot and Egger's test. However, this may be the small-study effect rather than true publication bias, especially in the presence of significant between-study heterogeneity [53]. We did attempt to adjust our quantitative analyses by including the missing studies. The trim and fill method is a statistical method used in meta-analysis that can underestimate the true positive effect when there is no publication bias, or can give less biased estimates when publication bias is present [54]. Using this conservative method, we found that the association between metformin and increased survival still remained. 


\section{ConCLUSION}

Overall, our meta-analysis demonstrated that metformin could be the drug of choice in patients with cancer and concurrent type 2 diabetes if there are no contraindications because it was associated with increased OS and CSS compared with other diabetic medications. Future prospective studies with larger sample sizes and alternate study design are required to confirm our findings.

\section{ACKNOWLEDGMENTS}

We thank Dr. Yousuke Nakai, Dr. Craig Currie, Dr. Peter Mazzone, and Dr. Tsung-Ming Chen for data coordination. We thank Dr. Mary Harris for language editing.

\section{Author Contributions}

Conception/Design: Ming Yin, Fahd Quddus, Provision of study material or patients: Ming Yin, Collection and/or assembly of data: Ming Yin, Jie Zhou, Fahd Quddus, Data analysis and interpretation: Ming Yin, Fahd Quddus, Manuscript writing: Ming Yin, Jie Zhou, Edward J. Gorak, Fahd Quddus

Final approval of manuscript: Ming Yin, Jie Zhou, Edward J. Gorak, Fahd Quddus

\section{DisClosures}

The authors indicated no financial relationships. Section editor: Powel Brown: None Reviewer "A": None

\section{REFERENCES}

1. Richardson LC, Pollack LA. Therapy insight: In fluence of type 2 diabetes on the development, treatment and outcomes of cancer. Nat Clin Prac 2005;2:48-53.

2. Giovannucci E, Harlan DM, Archer MC et al. Diabetes and cancer: A consensus report. Diabetes Care 2010;33:1674-1685.

3. Foretz M, Hebrard S, Leclerc J et al. Metformin inhibits hepatic gluconeogenesis in mice independently of the $\mathrm{kb} 1 / \mathrm{ampk}$ pathway via a decrease in hepatic energy state. J Clin Invest 2010;120:23552369 .

4. Kim YD, Park KG, Lee YS et al. Metformin inhibits hepatic gluconeogenesis through amp-activated protein kinase-dependent regulation of the orphan nuclear receptor shp. Diabetes 2008;57:306-314.

5. Staels B. Metformin and pioglitazone: Effectively treating insulin resistance. Curr Med Res Opin 2006;22 Suppl 2:27-37.

6. Bulcao C, Ribeiro-Filho FF, Sanudo A et al. Effects of simvastatin and metformin on inflammation and insulin resistance in individuals with mild metabolic syndrome. Am J Cardiovasc Drugs 2007; 7:219-224

7. Fidan $\mathrm{E}$, Onder Ersoz H, Yilmaz M et al. The effects of rosiglitazone and metformin on inflammation and endothelial dysfunction in patients with type 2 diabetes mellitus. Acta Diabetol 2011;48: 297-302.

8. Alimova IN, Liu B, Fan Z et al. Metformin inhibits breast cancer cell growth, colony formation and induces cell cycle arrest in vitro. Cell Cycle 2009;8: 909-915

9. Zakikhani M, Dowling R, Fantus IG et al. Metformin is an amp kinase-dependent growth inhibitor for breast cancer cells. Cancer Res 2006;66: 10269-10273.

10. Ben Sahra I, Regazzetti C, Robert G et al. Metformin, independent of ampk, induces mtor inhibition and cell-cycle arrest through redd1. Cancer Res 2011;71:4366-4372

11. Isakovic A, Harhaji L, Stevanovic D et al. Dual antiglioma action of metformin: Cell cycle arrest and mitochondria-dependent apoptosis. Cell Mol Life Sci 2007;64:1290-1302.

12. Liu J, Li M, Song B et al. Metformin inhibits renal cell carcinoma in vitro and in vivo xenograft. Urol Oncol 2013;31:264-270.

13. Luo $Q$, Hu D, Hu S et al. In vitro and in vivo antitumor effect of metformin as a novel therapeutic agent in human oral squamous cell carcinoma. BMC Cancer 2012;12:517.

14. Soranna D, Scotti L, Zambon A et al. Cancer risk associated with use of metformin and sulfonylure in type 2 diabetes: A meta-analysis. The Oncologist 2012;17:813-822.

15. Noto H, Goto A, Tsujimoto Tet al. Cancer risk in diabetic patients treated with metformin: A systematic review and meta-analysis. PloS One 2012;7 e33411.

16. Mazzone PJ, Rai H, Beukemann M et al. The effect of metformin and thiazolidinedione use on lung cancer in diabetics. BMC Cancer 2012;12:410.

17. Tan BX, Yao WX, Ge J et al. Prognostic influence of metformin as first-line chemotherapy for advanced nonsmall cell lung cancer in patients with type 2 diabetes. Cancer 2011;117:5103-5111.

18. Tierney JF, Stewart LA, Ghersi D et al. Practical methods for incorporating summary time-to-event data into meta-analysis. Trials 2007;8:16.

19. Lin DY, Sullivan PF. Meta-analysis of genomewide association studies with overlapping subjects. Am J Hum Genet 2009;85:862-872.

20. Duval S, Tweedie R. Trim and fill: A simple funnel-plot-based method of testing and adjusting for publication bias in meta-analysis. Biometrics 2000; 56:455-463.

21. Nobes JP, Langley SE, Klopper T et al. A prospective, randomized pilot study evaluating the ef fects of metformin and lifestyle intervention on patients with prostate cancer receiving androgen deprivation therapy. BJU Int 2012;109:1495-1502.

22. Patel T, Hruby G, Badani K et al. Clinical outcomes after radical prostatectomy in diabetic patients treated with metformin. Urology 2010;76 1240-1244.

23. Jiralerspong S, Palla SL, Giordano SH et al. Metformin and pathologic complete responses to neoadjuvant chemotherapy in diabetic patients with breast cancer. J Clin Oncol 2009;27:3297-3302.

24. Lee DJ, Kim B, Lee JH et al. The effect of metformin on responses to chemotherapy and surviva in stage iv colorectal cancer with diabetes. Korean J Gastroenter 2013;60:355-361.

25. Koo YX, Tan DS, Tan IB et al. Effect of concomi tant statin, metformin, or aspirin on rituximab treatment for diffuse large b-cell lymphoma. Leuk Lymphoma 2011;52:1509-1516.

26. Kumar S, Meuter A, Thapa P et al. Metformin intake is associated with better survival in ovarian cancer: A case-control study. Cancer 2013;119:555-562.

27. Currie CJ, Poole CD, Jenkins-Jones S et al. Mortality after incident cancer in people with and without type 2 diabetes: Impact of metformin on survival. Diabetes Care 2012;35:299-304

28. Kaushik D, Karnes RJ, Eisenberg MS et al. Effect of metformin on prostate cancer outcomes after radical prostatectomy. Urol Oncol 2013;June 27 [Epub ahead of print]

29. Lega IC, Austin PC, Gruneir A et al. Association between metformin therapy and mortality after breast cancer: A population-based study. Diabetes Care 2013;36:3018-3026.

30. Sadeghi N, Abbruzzese JL, Yeung SC et al. Metformin use is associated with better survival of diabetic patients with pancreatic cancer. Clin Cancer Res 2012;18:2905-2912.

31. Garrett CR, Hassabo HM, Bhadkamkar NA et al. Survival advantage observed with the use of metformin in patients with type ii diabetes and colorectal cancer. Br J Cancer 2012;106:1374-1378.

32. Romero IL, McCormickA, McEwen KA et al. Relationship of type ii diabetes and metformin use to ovarian cancer progression, survival, and chemosensitivity. Obstet Gynecol 2012;119:61-67.

33. HeX, Esteva FJ, Ensor J et al. Metformin and thiazolidinediones are associated with improved breast cancer-specific survival of diabetic women with her2 + breast cancer. Ann Oncol 2012;23:1771-1780.

34. Lee JH, Kim TI, Jeon SM et al. The effects of metformin on the survival of colorectal cancer patients with diabetes mellitus. Int J Cancer 2012;131 752-759.

35. Bayraktar S, Hernadez-Aya LF, Lei X et al. Effect of metformin on survival outcomes in diabetic patients with triple receptor-negative breast cancer Cancer 2012;118:1202-1211.

36. He XX, Tu SM, Lee MH et al. Thiazolidinediones and metformin associated with improved survival of diabetic prostate cancer patients. Ann Oncol 2012;22:2640-2645.

37. Chen TM, Lin CC, Huang PT et al. Metformin as sociated with lower mortality in diabetic patients with early stage hepatocellular carcinoma after radiofrequency ablation. J Gastroenterol Hepato 2011;26:858-865.

38. Nakai Y, Isayama H, Sasaki T et al. Clinical outcomes of chemotherapy for diabetic and nondiabetic patients with pancreatic cancer: Better prognosis with statin use in diabetic patients. Pancreas 2013;42:202-208.

39. Spratt DE, Zhang C, Zumsteg ZS et al. Metformin and prostate cancer: Reduced development of castration-resistant disease and prostate cancer mortality. Eur Urol 2013;63:709-716.

40. Hou G, Zhang S, Zhang Xet al. Clinical pathological characteristics and prognostic analysis of 1,013 breast cancer patients with diabetes. Breast Cancer Res Treat 2013;137:807-816.

41. Spillane SC, Bennett K, Sharp L et al. A cohort study of metformin exposure and survival in pa- 
tients with stage I-III colorectal cancer. Cancer Epidemiol Biomarkers Prev 2013;22:1364-1373.

42. Cossor Fl, Adams-Campbell LL, Chlebowski RT et al. Diabetes, metformin use, and colorectal cancer survival in postmenopausal women. Cancer Epidemiol 2013;37:742-749.

43. Sandulache VC, Hamblin JS, Skinner HD et al. Metformin use is associated with improved survival in patients with laryngeal squamous cell carcinoma. Head Neck 2013;June 19 [Epub ahead of print].

44. Zhang F, Yang Y, Skrip L et al. Diabetes mellitus and risk of prostate cancer: An updated meta-analysis based on 12 case-control and 25 cohort studies. Acta Diabetol 2012;49 Suppl 1:235-246.

45. Rubenstrunk A, Hanf R, Hum DW et al. Safety issues and prospects for future generations of ppar modulators. Biochim Biophys Acta 2007;1771: 1065-1081.

46. Tseng SC, Huang YC, Chen HJ et al. Met- formin-mediated downregulation of p38 mitogen-activated protein kinase-dependent excision repair cross-complementing 1 decreases DNA repair capacity and sensitizes human lung cancer cells to paclitaxel. Biochem Pharmacol 2013;85: 583-594.

47. Iliopoulos D, Hirsch HA, Struhl K. Metformin decreases the dose of chemotherapy for prolonging tumor remission in mouse xenografts involving multiple cancer cell types. Cancer Res 2011;71: 3196-3201.

48. Dowling RJ, Zakikhani M, Fantus IG et al. Metformin inhibits mammalian target of rapamycin-dependent translation initiation in breast cancer cells. Cancer Res 2007;67:10804-10812.

49. Chen HP, Shieh JJ, Chang CC et al. Metformin decreases hepatocellular carcinoma risk in a dosedependent manner: Population-based and in vitro studies. Gut 2013;62:606-615.
50. Kalender A, Selvaraj A, Kim SY et al. Metformin, independent of ampk, inhibits mtorc1 in a rag gtpase-dependent manner. Cell Metab 2010;11 390-401.

51. Blandino $G$, Valerio $M$, Cioce $M$ et al. Metformin elicits anticancer effects through the sequential modulation of dicer and c-myc. Nature Comm 2012;3:865.

52. Bowker SL, Majumdar SR, Veugelers $P$ et al. Increased cancer-related mortality for patients with type 2 diabetes who use sulfonylureas or insulin. Diabetes Care 2006;29:254-258.

53. Terrin N, Schmid CH, Lau J et al. Adjusting for publication bias in the presence of heterogeneity. Stat Med 2003;22:2113-2126.

54. Peters JL, Sutton AJ, Jones DR et al. Performance of the trim and fill method in the presence of publication bias and between-study heterogeneity. Stat Med 2007;26:4544-4562.

CME This article is available for continuing medical education credit at CME.TheOncologist.com.

EDITOR'S NOTE: See the accompanying commentary on pages 1245-1247 of this issue. 